\title{
Correction to: Modeling interactions between saturated and un-saturated zones by Hydrus-1D in semi-arid regions (plain of Kairouan, Central Tunisia)
}

\author{
Mariem Saâdi • Adel Zghibi • Sabri Kanzari
}

Published online: 3 October 2018

C) Springer Nature Switzerland AG 2018

Correction to: Environ Monit Assess (2018) 190: 170 https://doi.org/10.1007/s10661-018-6544-3

Figure 3 in this article has been obscured as the required permissions to reproduce it were not obtained.

The online version of the original article can be found at https://doi. org/10.1007/s10661-018-6544-3

M. Saâdi · A. Zghibi $(\bowtie)$

Faculté des Sciences de Tunis, UR13ES26 Paléo-environnement, Géomatériaux et Risques Géologiques, Université de Tunis El Manar, 2092 Tunis, Tunisie

e-mail: adelzguibi@yahoo.fr

e-mail: adel.zghibi@fst.utm.tn

S. Kanzari

Institut National de Recherche en Génie Rural, Eau et Forêts, Rue Hédi EL Karray El Menzah IVBP 10Ariana 2080, Tunisie 